\title{
Exploring context-awareness for ubiquitous computing in the healthcare domain
}

\author{
Jesper Kjeldskov $\cdot$ Mikael B. Skov
}

Received: 31 January 2006/ Accepted: 23 May 2006/Published online: 7 November 2006

(C) Springer-Verlag London Limited 2006

\begin{abstract}
Ubiquitous technologies have potentials to play major roles in different real world organizational settings. One of the areas where applying ubiquitous technologies has been given a lot of attention is in the healthcare domain. Here, users are frequently on the move while at the same time relying increasingly on centralized computerized information. In this paper, we explore ubiquitous technologies in the real world through two studies in the healthcare domain. First, we look at the use and usability of a ubiquitous electronic patient record (EPR) system distributed on desktop and laptop computers throughout a large hospital. Secondly, we present an extension to this ubiquitous computing environment in the form of a context-aware mobile computer terminal prototype. The usability of the mobile EPR prototype was evaluated in both laboratory and field settings. Our results indicate that the usefulness of a ubiquitous computing environment supporting work activities in healthcare can benefit from context-aware mobile information access. However, interaction design for such systems must be carefully thought out and thoroughly evaluated. Also, while the use of mobile and stationary computers complement each other very well, we found that the usefulness of ubiquitous computing environments in healthcare may benefit from additional elements such as situated displays at
\end{abstract}

J. Kjeldskov $(\bowtie) \cdot$ M. B. Skov

Department of Computer Science, Aalborg University, Fredrik Bajers Vej 7E, 9220 Aalborg East, Denmark

e-mail: jesper@cs.aau.dk key locations and on key objects, and from seamless integration between the different devices comprising the system as a whole.

\section{Introduction}

Over the past years, emerging computer technologies have drawn enormous attention as they often yield new and innovative use in work as well as in leisure. We are currently on the move away from traditional desktop-based computer technologies towards ubiquitous computing environments that will potentially enfold us in almost all of everyday situation and activity. We encounter these computing environments everywhere: in our homes, cars, work places, shops, restaurants, cinemas etc., and thus, such computing environments have to accommodate several different use situations and user groups. Consequently, ubiquitous computing environments have received immense attention from both academia and industry, in order to explore their promising opportunities, apparent limitations, and experienced implications for interaction design. Because the use of ubiquitous computing environments is often closely related their contextual settings, one of the avenues of research which has concerned human-computer interaction researchers and practitioners is the ability of ubiquitous computing environments to explore context-awareness in interaction design.

In this paper, we present our experiences with two ubiquitous computing environments to support nurses and doctors in conducting their work activities within a healthcare domain. First, we explore the use of an 
existing ubiquitous computing environment at a large hospital in the form of a commercial EPR system, distributed on a series of desktop and laptop based computers throughout the organization. Second, we explore the design and use of and an experimental ubiquitous computing environment extending the existing ensemble of technologies with a mobile context-aware component facilitating a higher degree of pervasive and nomadic use.

This paper is structured in the following way. First, we discus a series of issues concerning emerging ubiquitous technologies and present some of the current experiences with information technologies in healthcare reported in the literature. Secondly, we present our initial usability study of a ubiquitous computing environment in healthcare. The findings from this study are then outlined as a series of highlevel themes describing problems and advantages encountered during use. Third, we present the design of an experimental context-aware, mobile, EPR system, supplementing the existing ubiquitous computing environment. Fourth, we show how this prototype system was evaluated in a laboratory as well as in field settings at the hospital, and outline the primary findings from these evaluations. Finally, we discuss the findings from the evaluations of the mobile EPR component prototype in the light of the themes identified in our initial study and discuss contextaware interaction design for ubiquitous computing environments.

\section{Related work}

The diversity of users and use situations makes it challenging and difficult to design ubiquitous computing environments. Designers have to pay attention to several issues in the use domain if the computing environment is to become useful and successful. Furthermore, only limited practical experiences with the design and use of ubiquitous computing environments for the real world are reported in the literature.

\subsection{Ubiquitous computing environments and usability}

As instances of ubiquitous technologies, distributed terminals, mobile and handheld technologies etc. have the potentials to serve major roles in different organizational contexts in the future because they will provide users the ability to access information and services when away from their desktop [7]. Within a multitude of work domains, this may potentially lead to new ways of working, as people will be able to conduct or perform work activities different from what they are presently able to. However, designing and implementing these types of information systems will also be highly challenging and difficult. Research studies show that ubiquitous and mobile technologies continue to challenge our existing body of knowledge on analysis, design, implementation and evaluation of information systems [3, 6]. While such challenges are not unique for any specific emerging technologies [20], ubiquitous, and mobile technologies may exhibit novel and unprecedented complexity for interaction design as user interaction with such technologies will be continuous and pervasive [3].

It is generally considered of great importance for designers to consider the future use situation when designing and implementing software information systems. Naturally, this is also true for ubiquitous computing environments. However, for ubiquitous computing environments, the traditional focus of effectiveness and efficiency of software use may not be applicable, suitable, or desirable in the same way as we are used to. Instead, designers have to look broadly at the ensemble of activities in the physical world (e.g. work activities), interactions between people in the physical world, and their use of technology. Thus, some of the key research problems in relation to the design of ubiquitous computing environments have come to understand the everyday character of the environment [6] and to design systems that conform, not disrupt, the natural workflow of the user [5]. Few studies provide suggestions on how to achieve a smooth interaction between the user and a ubiquitous computing environment. Exceptions count, for example, Barkhuus and Dey [3], who examine three levels of interactivity for context-aware mobile systems.

Rubin [18] argues that usability evaluation of software systems is an efficient and well-documented approach for understanding and classifying the interaction between a user and a software system. Usability evaluations can be utilized to identify problems in the interaction design, and can potentially inform designers about the extent to which their software product is useful [14]. In recent years, evaluating the usability of emerging technologies has also become vital from a business perspective as indicators of potential success or failure of new technologies, within an area typically associated with considerable financial investments and risk for technology providers and manufacturers [16], [18]. Combining this with the growing complexity of new technology, identifying fundamental usability problems with, for example, ubiquitous computing 
environments may prove significant for informing successful design and implementation of such systems. Furthermore, as users become more diverse in terms of, for example, skills, motivation, and experience, obtaining a high level of usability of new technologies becomes a substantial challenge to designers and businesses, asthey have to accommodate this great diversity.

\subsection{Ubiquitous computing in healthcare}

Ubiquitous computing environments can potentially influence and change work practices within a multitude of different settings in the healthcare domain dramatically. Healthcare work for example, in hospitals, is typically characterized by very complex and specialized procedures in which information technology may contribute to improved performance, reduction of errors made in treatment of patients, reduction of economical costs, etc. Different types of software systems are currently being introduced in many hospitals and other parts of the healthcare domain. Typically, these systems are connected through various types of computer networks and are widely dispersed throughout the physical organization. Hence, they can be classified as ubiquitous computing environments.

Of particular interest, a lot of resources are being put into implementation and use of EPR systems. EPR systems collect information about the history of treatment performed on the patients admitted to a hospital. The hospital personnel use the patient record to diagnose diseases, and to document and coordinate treatment. Within the last 20 years, a considerable amount of effort has been devoted to the development of EPR systems. The primary motivation for this effort is that unlike paper-based patient records, EPR will be accessible to all relevant persons, independent of time and location.

The design of EPR systems is a huge challenge for our community, raising a wide range of still unanswered questions related to issues such as screen layout, interaction design, and integration into work processes. Where should the systems be located and who should enter the data? How do we make sure that input is complete and accurate? How are the different work processes in healthcare structured and coordinated? What is the most useful way of displaying and accessing the vast quantity of patient data [4] ? In the light of these questions, a lot of research has been published in the literature about EPR systems and how to meet challenges related to design and use of computer system in healthcare. Specifically, much attention has been given to issues such as information sharing [8], support for cooperation [11] and privacy [17]. While much of this research is based on studies on the use of traditional paper-based patient records, suggesting viable electronic counterparts, however, little research has been published based on studies that inquire into the use of the mass of EPR systems already in use.

In 2002, the Danish government decided that all Danish hospitals must replace the traditional paperbased patient records with by 2005 . However, it was up the regional authorities to decide on the details of deployment. Thus, a number of projects were conducted within this period of time with the aim of developing and evaluating EPR systems (see for example the work of Bardram et al. in [2]). In relation to a regional Danish research program entitled "The Digital Hospital", we have studied the use and usability of a commercial EPR system currently constituting the backbone of a ubiquitous computing environment at a large regional hospital (IBM IPJ 2.3). In addition to this, we have designed, implemented and evaluated an experimental mobile EPR prototype terminal, extending the current system's functionality and scope. Driving this study, we were concerned with the following research questions:

1. What challenges and potentials characterize the use and usability of the ubiquitous EPR system currently in use at the hospital?

2. How can the use and usability of the ubiquitous EPR system be improved through context-aware mobile information access?

The following sections describe the details of our initial usability evaluations of the EPR system currently in use and subsequent experimental design process of a mobile context-aware counterpart.

\section{Study A: ubiquitous EPR in the real world}

As our point of departure, we conducted a longitudinal usability evaluation of IBM's EPR system IPJ 2.3 currently in use throughout the Hospital of Frederikshavn, Denmark. The aim of study A was to inquire into the use and usability of ubiquitous EPR in relation to carrying out typical work activities at a regional hospital prior to national implementation of such systems. The two main screens of the evaluated EPR system are illustrated in Figs. 1, 2 and 3.

\subsection{Method}

The evaluation of the EPR system was carried out in a dedicated usability laboratory at Aalborg University, 
Fig. 1 Ubiquitous EPR use in the healthcare domain

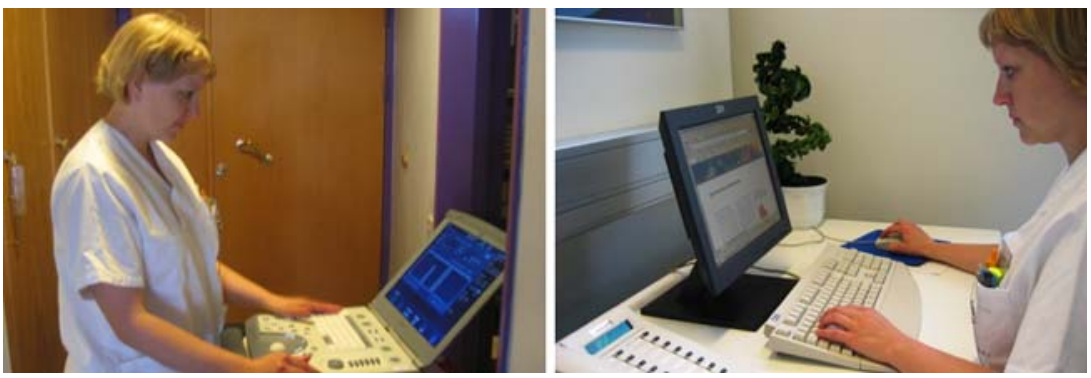

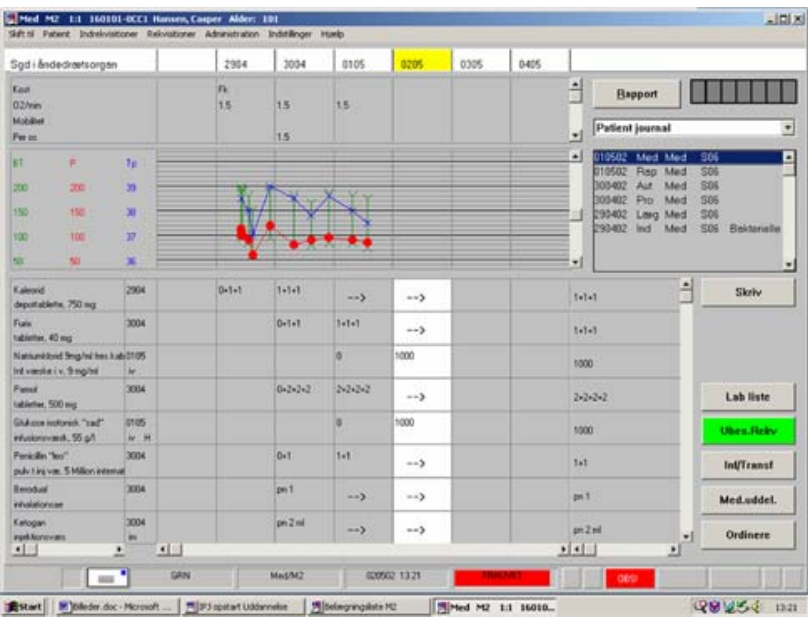

Fig. 2 Screen shot from IPJ 2.3 illustrating the primary information, for example, previous measured temperatures, heart rates, on a specific patient

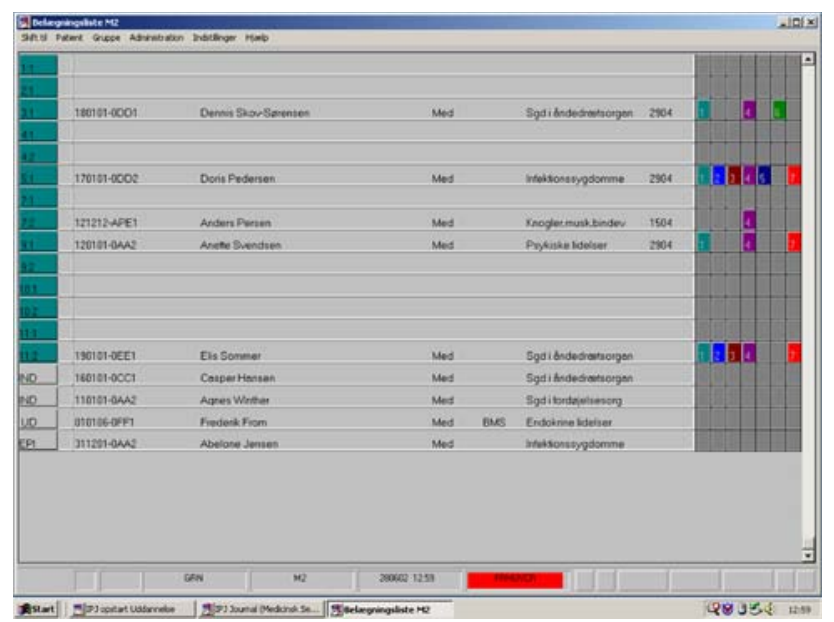

Fig. 3 Screen shot from IPJ 2.3 showing the central list of patients on the ward with overall information about diagnosis and treatment

over a period of little more than one year. In order to avoid just getting a snapshot of the use and usability of the system, we conducted a longitudinal study, involving two separate evaluations with the same users.
The first evaluation was conducted over two days in May 2002, shortly after the system had been taken into use at the hospital. The second evaluation was conducted over two days in August 2003, after the system had been used extensively in daily work at the hospital for more than a year. In total, 16 evaluation sessions of approximately $1 \mathrm{~h}$ were carried out.

The evaluation involved two parts:

1. A think-aloud evaluation with the purpose of identifying usability problems in the EPR system.

2. A series of interviews with the purpose of gaining insight into the integration of the EPR with the work of the nurses.

The details of the evaluation are described below.

\subsubsection{Preparation}

Prior to the first evaluation, the research team inquired thoroughly into the work activities at the hospital related to the use of patient records. This was done through observations at the hospital and interviews with key personnel. At the same time, the research team also investigatedthe specific functionality of the EPR system to be evaluated. Based on this, the research team and key personnel at the hospital assigned to the implementation of the EPR System in the organization developed a series of use scenarios and a number of tasks for the evaluation in collaboration. The use scenarios and tasks went trough several iterations of pilot testing and modification before the evaluation took place.

\subsubsection{Test subjects}

The evaluation involved eight trained nurses from the Hospital of Frederikshavn. All nurses were women, aged between 31 and 54 years with professional work experience ranging from 2 to 31 years (at the time of the first evaluation). Prior to the first evaluation, all nurses had attended a course on the IPJ 2.3 system amounting to between 14 and $30 \mathrm{~h}$ and were just 
beginning to use the system in their daily work. They characterized themselves as novices or beginners in relation to the use of IT in general. At the time of the second evaluation, they had all used the system extensively in their daily work for more than a year and now characterized themselves as system experts. All test subjects participated in both the 2002 and the 2003 evaluations except for one person who had left the hospital in the meantime. As a replacement, the hospital found another nurse who matched the characteristics of the original participant.

\subsubsection{Tasks}

The purpose of the usability evaluations was to inquire how the EPR system supports typical work activities at the hospital. Based on our scenarios, we designed three tasks with a number of sub tasks centered on the core purpose of the system-such as retrieving information about patients, registering information about treatments, making treatment notes, and entering measurements.

\subsubsection{Procedure}

The evaluation sessions consisted of two parts: (1) a hands-on part where the nurses used the system to solve the assigned tasks, and (2) a semi-structured interview. The hands-on part of the evaluation was based on the think-aloud protocol as described in [14, $16,18]$. If a test subject had problems with a task and had tried all the options she could identify, the test monitor provided her with help to find the solution. If a test subject was completely unable to solve a task, the test monitor asked her to go on to the next task. The hands-on sessions lasted approximately $45 \mathrm{~min}$. After the hands-on sessions, four randomly selected nurses were interviewed about their work and their opinions about the system as well as its integration in and influence on their work. The interviews were semistructured, based on a list of questions and a number of issues that could be raised. The evaluation sessions were conducted over 2 days. One of the authors of this article was a test monitor throughout all test sessions. The other author conducted the interviews in collaboration with a third researcher.

\subsubsection{Setting}

The evaluations were all conducted in a state-of-the-art usability laboratory at Aalborg University, facilitating close-up observation of the test subject's interaction with the evaluated system. This involved the use of three different rooms: a subject room, an observation room and a control room. For the EPR evaluation, the subject room was equipped with a standard PC with a 19 in. screen and a standard mouse and keyboard, matching the hardware used at the hospital. During the hands-on sessions, the test monitor observed the test subjects, encouraged them to think aloud and asked questions for clarification. Two additional researchers operated the video equipment and took notes in the separate control and observation rooms, respectively.

\subsubsection{Data collection}

All evaluation sessions were recorded on digital video. Two remotely controlled motorized cameras captured overall and close-up views of the test subject and test monitor. The image on the PC screen was converted to composite video and mixed with the camera images to one composite video signal. The video recordings also contained audio tracks with the verbal utterances of the test subject and test monitor.

\subsubsection{Data analysis}

Following the evaluations a log file was produced from each test. Each of the three individual researchers then used the video recordings and these $\log$ files as empirical foundation for producing three individual lists of usability problems. These lists were then merged into one. The severity of the identified problems was rated as critical, serious or cosmetic, based on the guidelines proposed by Molich in [14]. The rating was done individually and followed by negotiation in cases of disagreement.

\subsection{Findings from the evaluation of IPJ 2.3}

The evaluation identified a substantial amount of usability problems with the current EPR system. The total number of identified usability problems was 75 , distributed on 9 critical, 39 serious and 27 cosmetic problems. The 75 usability problems were related to various concerns of human-computer interaction, e.g. problems with finding and storing information, problems related to high complexity of screen layouts and problems with inconsistent interaction design. The 75 identified usability problems are described in detail in [12]. At the same time, the usability test also identified a number of strengths in the interface of the EPR system, for example, easy registration of values, good integration with some existing work activities and good overview of the patients in the ward. 
While the identified strengths and weaknesses provided valuable information for redesign and focused training, the usability problems by themselves provided little input for breaking out of the boundaries of the existing system. In order to do so, we took a step back from the list of usability problems and conducted a grounded analysis of observed interactions with the system and the statements from the interviews. From the grounded analysis, we identified three themes reflecting more abstract concerns of the usability of EPRs:

1. Mobility

2. Complexity

3. Relation to work activities

These are described more in detail, in the following.

\subsubsection{Mobility}

During the usability evaluations and in the interview afterwards, most nurses would stress concerns about being mobile while working with the system. To meet this challenge, the use of laptop computers rather than desktop workstations had been suggested and discussed at the hospital. However, most of the nurses stated that they would find it impossible or unfeasible to carry a laptop computer around the ward every time they were to conduct work tasks away from their office. One problem was the size and weight of the laptop, as they would also have to carry other instruments. As it turned out later, the issue of mobility was so immense that the hospital implemented a semi-mobile way of accessing the EPR system through laptops connected to a wireless network being wheeled around the hospital wards on trolleys.

\subsubsection{Complexity}

Another overall concern or problem was the complexity and fragmentation of information. Most nurses found it difficult to locate the necessary patient information as they were solving tasks. This sometimes led to inadequate or incomplete task completion. Hence, the nurses would feel insecure whether they had found the right information and whether they had succeeded in finding all relevant information.

\subsubsection{Relation to work activities}

Most nurses experienced problems with the use of the EPR system because they had difficulties relating the information in the system to their work activities.
The problem was that they would typically use different kinds of information in real life to decide how to solve a problem, for example, visible conditions of a patient. Another concern related to the fact that the system only partially reflected the current work task, making it difficult for the test subjects to find or store information.

\section{Study B: an experimental mobile EPR system}

Motivated by the findings from our evaluation of the EPR system, we carried out a field study, on the work activities at the Hospital of Frederikshavn related to the use of EPR in practice. In concert with the findings from the usability evaluation outlined above, this suggested a design solution with the following characteristics.

1. Supporting the highly mobile work activities of nurses by being mobile /handheld.

2. Reducing complexity by adapting to its context: location, time, tasks.

3. Eliminating double registering of information (first written down on paper and then entered into the desktop or laptop PC later) by being integrated with the existing patient record system.

While facilitating access to patient information at the 'point of care' is not a new idea [1, 15, 22], adapting information and functionality in a mobile EPR system to its context is a novel approach to improve the interaction design of such systems that has not yet been investigated thoroughly. On the basis of the findings from the usability evaluation and subsequent field study, an experimental prototype of a handheld context-aware EPR system for supporting the morning procedure, MOBILEWARD, was designed and implemented [9, 10]. The system is described briefly as follows; a more detailed description can be found in [21].

\subsection{Architecture}

MOBILEWARD was designed for a series of Compaq iPAQ 3630 running the Microsoft ${ }^{\circledR}$ PocketPC operating system. The system uses a wireless local area network (wLAN) for network communication. The system was implemented in Microsoft embedded Visual Basic 3.0. For the first experimental prototype of MOBILEWARD, context-awareness was simulated by means of a "context control center" application. The control center runs on a separate iPAQ connected to the wireless network. Through this application, an 
Fig. 4 Interface layout from MOBILEWARD illustrating patients admitted the hospital (left) and information on a selected patient (right)

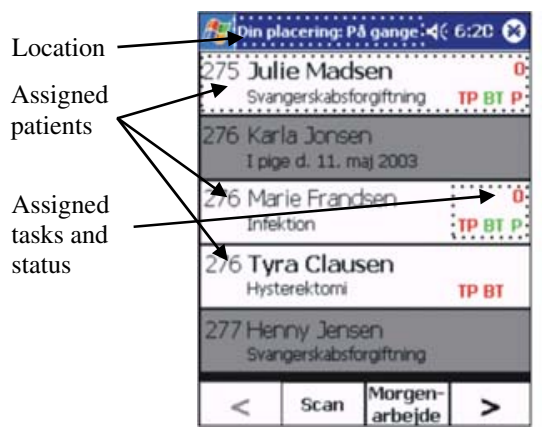

operator can trigger "context events" in MOBILEWARD simulating that the user has entered a specific room, scanned the barcode on a specific patient etc. This approach was chosen to facilitate early evaluation of the experimental design solution without having to worry about the technical challenges of context sensing at the hospital before this had proven to be a viable approach from the user's perspective. In later versions of the system, real sensing of the environment can be implemented where found promising.

For discussions on how to sense environments, see, for example, [3] and [19].

\subsection{Interface design}

MOBILEWARD is designed to support work tasks during morning procedure at the hospital ward. The design is based on two basic concepts. First, the system is designed to reflect the context of the user in the sense that it is able to sense and react to a number of changes in the environment. Second, as the use of a pen for typing in the information would sometimes be inappropriate because the nurses would often use the system while being mobile or engaged in other activities, the interface design incorporates a visual layout with large-scale buttons that enables finger-based interaction through the touch-screen of the iPAQ.
MOBILEWARD is context-aware in the sense that the system recognizes the location of the nurse and presents information and functionality accordingly. Before visiting assigned patients, the nurses often want to get an overview of the specific information about each patient, for example, previous measured values. This typical takes place at the nurse's office or in the corridor. The windows related to these locations are shown in Fig. 4.

When located in the corridor, the system by default displays all the patients admitted to the ward. The patient list is ordered by ward number. Patients assigned for morning procedure are shown with a white background and the names of patients assigned to the nurse using the system are boldfaced (e.g. "Julie Madsen" and "Tyra Clausen" on Fig. 4). At the top of all windows, the nurse can see her current physical location as interpreted by the system. In the example on Fig. 4, the nurse is in the corridor. For each patient, MobileWARD provides information about previous tasks, upcoming tasks and upcoming operations. The indicators TP (temperature), BT (blood pressure) and $\mathrm{P}$ (pulse), show the measurements that the nurse has to perform. The indicators are either presented with red text (value still to be measured) or green text (value already measured). Above the three indicators, an " $\mathrm{O}$ " indicates an upcoming operation (within $24 \mathrm{~h}$ ), which
Fig. 5 Screens displayed in the ward in relation to the tasks of measuring temperature, blood pressure, and pulse
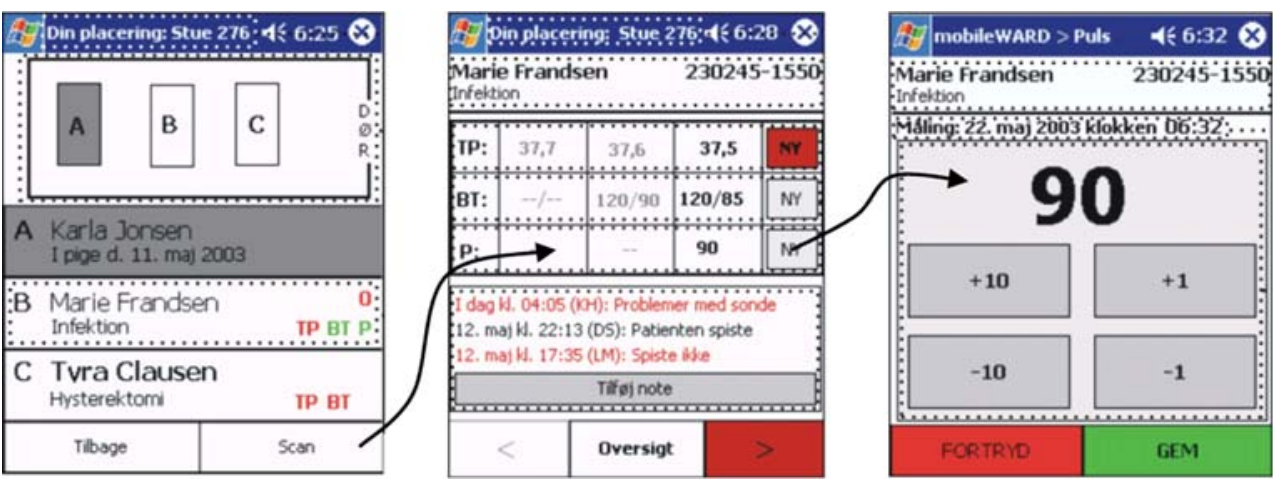
usually requiresthe patient to fast and be prepared for operation.

If the nurse wants to view data about a specific patient, she can click on one of the patients on the list. This will open the window shown on Fig. 4 (right), displaying the name and personal identification number of the patient, the previous two sets of temperature, blood pressure, and pulse measurements taken, as well as written notes regarding the treatment of the patient. This window is accessible at any time and location. Thus, the nurse can choose to look up more specific details about each patient while located in the corridor or in the office. In order to enter new data into the system, the nurse has to scan the barcode identification tag on the patient's wristband, using the "scan" function in the bottom of the screen. This is described further below.

The aim of this design is to provide the nurse with information that helps her plan the scheduled morning procedure. The system presents information and functionality adapted to the location of the nurse and the time of the day. Furthermore, the system knows the status of each patient and represents already measured values and values yet to be measured by simple color codes.

When the nurse enters a ward, the system automatically displays a different set of information. This is illustrated in Fig. 5. At the top of the screen, the nurse can see her physical location as interpreted by the system (e.g. ward 276). Below this, information about the patients on the current ward is presented, resembling the information available on the patient list displayed in the corridor, with the addition of a graphical representation of the physical location of the patient's respective beds. In this way, MOBILEWARD aims at presenting only relevant information to the nurse, e.g. by excluding patients from other wards. Like in the corridor, data about the patients is available by clicking on their names (or on their bed-icon). At the bottom of the screen, the nurse can activate the barcode scanner ("scan") used to identify a patient, prior to entering data into the system.

After having scanned a patient, the nurse can type in measured values (Fig. 5, center). This window shows previous measurements of values and provides functionality for typing in new values. By clicking the new value button ("ny"), the system displays a window for entering new values (Fig. 5, right). Below the personal information (name and personal identification number), date and time is shown. In the gray box, the nurse can input the measured value by editing the shown value. This is done, by pressing the large sized buttons on the screen with a finger. The number shown by default is the latest measurement. The reason for this is that the latest measure is most likely to be close to the newest one. If there is a huge difference, this is clearly signaled to the nurse, as she will have to perform more button presses than usual, providing an implicit opportunity to see whether, e.g. the temperature for a given patient is rising or falling. The Save button stores the value in the database along with the date, time, and user identification. Furthermore, the system updates the status of the task as having been carried out.

\subsection{Usability evaluation of MOBILEWARD}

We evaluated the studies on the use of the MOBILEWARD prototype through two separate studies conducted in the laboratory and in the field, respectively. The first study was conducted in our usability laboratory with the objective to evaluate MOBILEWARD in a controlled environment where we could assign the nurses to specific tasks and closely observe their use of the system. In addition to this, we also wantedthe laboratory evaluation to involve mobility and context. In order to achieve this, we modified the standard laboratory setup in a number of ways. The second study took place at the Hospital of Frederikshavn. The aim of this evaluation was to study the usability of MOBILEWARD for supporting real work activities at a hospital setting involving real nurses and real hospitalized patients. In order to achieve this, we adopted an observational approach combined with questions for clarification while the nurses were not directly engaged in conducting their work. The details of the two studies are described below.

\subsubsection{Setting}

The usability laboratory was set up to resemble a part of the physical space of a hospital department (Fig. 6). This included the use of two separate evaluation rooms connected by a hallway. Each of the evaluation rooms was furnished with beds and tables similar to real hospital wards. From a central control room, the evaluation rooms and the hallway could be observed through one-way mirrors and via remotely controlled motorized cameras mounted in the ceiling.

The field evaluation was carried out at the Medical Department at the Hospital of Frederikshavn (Fig. 7). This included the physical area of seven hospital wards, an office with reception, a rinse room and a break-out area connected by a central hallway and involved nurses at work and patients committed to the hospital. 


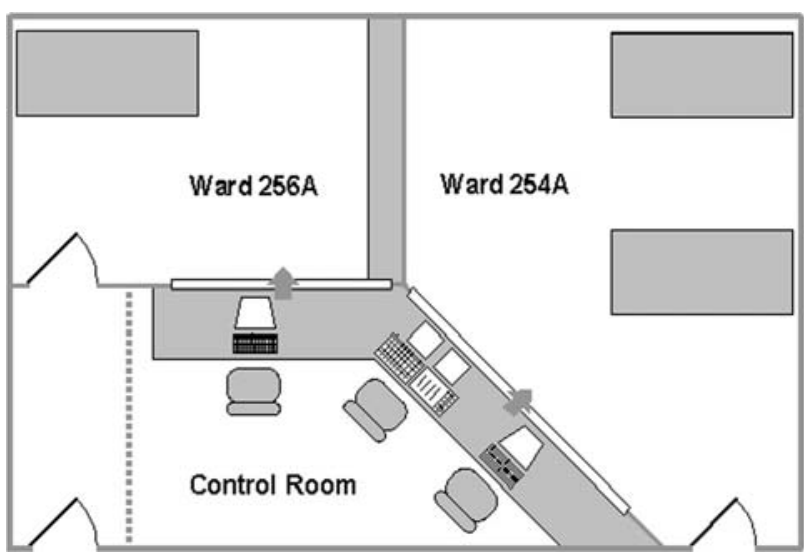

Fig. 6 Physical layout of the lab set up to emulate a section of the hospital ward

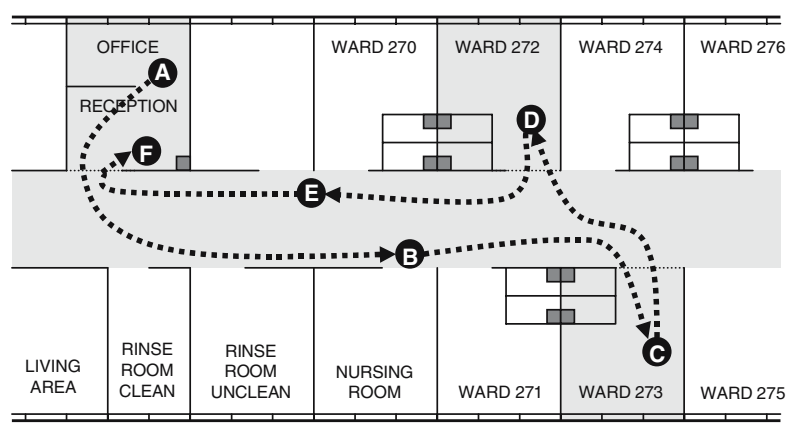

Fig. 7 Physical layout of the hospital ward used for the field evaluation

\subsubsection{Data collection}

High quality audio and video data from the laboratory and field evaluations were recorded digitally. In the laboratory, a tiny wireless camera was clipped on to the mobile device (Fig. 8), providing us with a close-up view of the screen and user-interaction. This was then merged with video signals from the ceiling-mounted cameras (Fig. 9).

Motivated by the challenges of capturing highquality video data during usability evaluations in the field, we designed and built a portable configuration of audio and video equipment to be carried by the test subject and an observer, allowing a physical distance of up to 10 meters between the two. The configuration consists of a tiny wireless camera (also used in the laboratory evaluation described above) clipped-on to the mobile device (Fig. 8) and a clip-on microphone worn by the test subject. Audio and video is transmitted wireless to recording equipment carried by the observer (Fig. 10). In the test monitor's bag, the video signal from the clip-on camera can be merged with the video signal from a handheld camcorder (Picture-in-Picture) and recorded digitally. This allows us to record a high-quality close-up view of the screen and user-interaction as well as an overall view of user and context. During the evaluation, the observer can view the user's interaction with the mobile device on a small LCD screen and monitor the sound through earphones. For ethical reasons, we were not permitted to film the hospitalized patients.

\subsubsection{Test subjects}

Twelve test subjects participated in the evaluations. Six test subjects (four females and two males) aged between 28 and 55 years participated in the laboratory study whereas six test subjects (all females) aged between 25 and 55 years participated in the field evaluation. All test subjects were trained nurses employed at a large regional hospital and had between 2 and 36 years of professional experience. All subjects were mobile phone users but novices with the use of handheld computers. All test subjects were frequent users of a stationary EPR system and described themselves as experienced or semi-experienced users of IT.

\subsubsection{Tasks}

All test subjects in the laboratory were given tasks to solve while using the system. The tasks were derived from an ethnographic study at a hospital ward and covered the duties involved in conducting standard morning work routines. This involved (1) checking up on a number of assigned patients based on information in the system from the previous watch, (2) collecting and reporting scheduled measurements such as temperature, blood pressure, and pulse, and (3) reporting anything important for the ongoing treatment of the patients should be taken into consideration on the next shift. The field evaluation did not involve any researcher control in form of task assignments but was structured by the work activities of the nurses in relation to conducting standard morning work routines.

\subsubsection{Procedure}

Before the evaluation sessions, the test subjects were given a brief introduction to the system. This included the room-sensing functionality and the 
Figs. 8-9 8 Wireless miniature camera mounted on PDA. 9 Video recording from usability evaluation

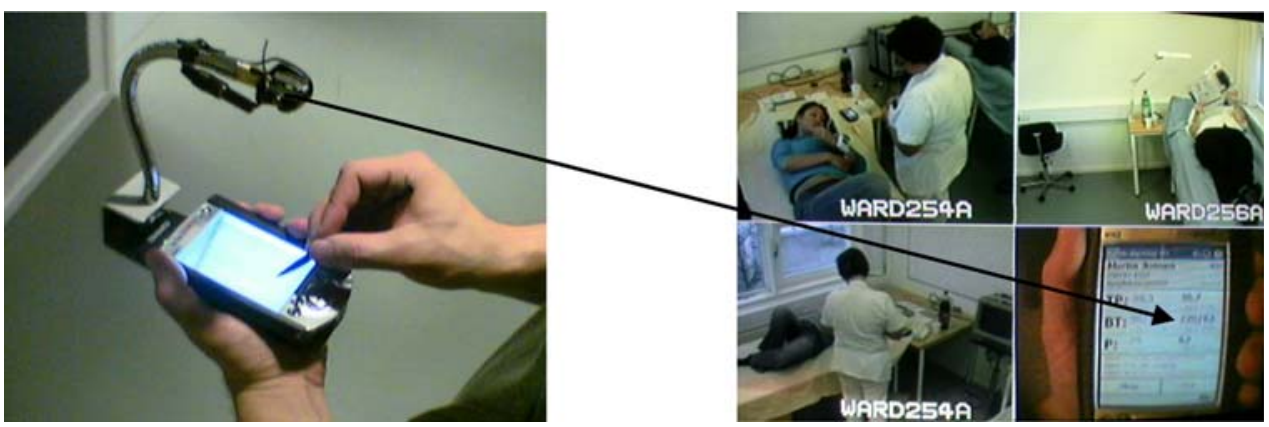

procedure for scanning patients' bar-code tags. The test subjects were also instructed on how to operate the available instruments for measuring temperature, blood pressure and pulse. In the lab sessions, the evaluation sessions were structured by the task assignments. The tasks required the test subjects to interact with all three patients in the two hospital wards, and to move between the two rooms through the connecting hallway a number of times. The nurses were encouraged to think aloud throughout the evaluation, explaining their comprehension of and interaction with the system. The lab evaluations lasted between 20 and $40 \mathrm{~min}$. In the field sessions, the evaluation sessions were not structured by tasks but by the actual work activities of the nurses. This involved interaction with three patients in different wards and moving between different rooms through the connecting hallway a number of times. The nurses were encouraged to think aloud when possible. The evaluations lasted $15 \mathrm{~min}$ on average. In order to be able to include a suitable number of nurses, the field evaluation took place over 2 days. After all evaluation sessions, the test subjects filled out a brief questionnaire.

\subsubsection{Roles}

Each evaluation session involved six people. One nurse used the system for carrying out the assigned tasks or real work activities depending on the experimental setting (laboratory or field). One researcher acted as test monitor and asked questions for clarification. A second researcher operated the context-control centre and the video equipment. In addition, each evaluation session involved either three students acting as hospitalized patients (in the laboratory) or three real hospitalized patients in their beds (in the field). Due to the real-life nature of the study that took place at the hospital, each field evaluation session involved different patients.

\subsubsection{Data analysis}

The data analysis aimed at creating two independent lists of usability problems identified from the two experimental settings. The usability problems were classified as cosmetic, serious or critical, according to Molich [14]. The two usability evaluations amounted to approximately $6 \mathrm{~h}$ of video recordings depicting the 12 test subjects' use of the system. All sessions were analyzed in random order by two teams of two evaluators where each team analyzed the videos in a collaborative effort, allowing immediate discussions of identified problems and their severity. As a guideline for the collaborative analysis, each identified usability problem would be discussed until consensus had been reached. The two teams produced two lists of usability problems. Subsequently, these were merged into one complete list. Again, this was done in a collaborative effort, discussing each problem and severity until consensus had been reached.

\section{Findings and discussion}

We identified a total of 37 different usability problems from the 12 laboratory and field sessions. Eight problems were assessed to be critical, 19 problems were assessed to be serious, and 10 problems were assessed to be cosmetic. Our study showed that the laboratory setting revealed more usability problems than the field setting. The six test subjects in the lab experienced 36 of the 37 usability problems whereas the six test subjects in the field setting experienced 23 of the 37 usability problems. Fourteen usability problems (one critical, nine serious, four cosmetic) were unique to the lab setting, whereas one serious usability problem was unique to the field. Regarding the identified critical problems, the lab setting identified all eight critical problems and the field setting identified seven critical problems. Considering the serious problems, the lab 
Fig. 10 Observer (left) carrying and operating portable audio/video equipment (right) for capturing high-quality data in the field
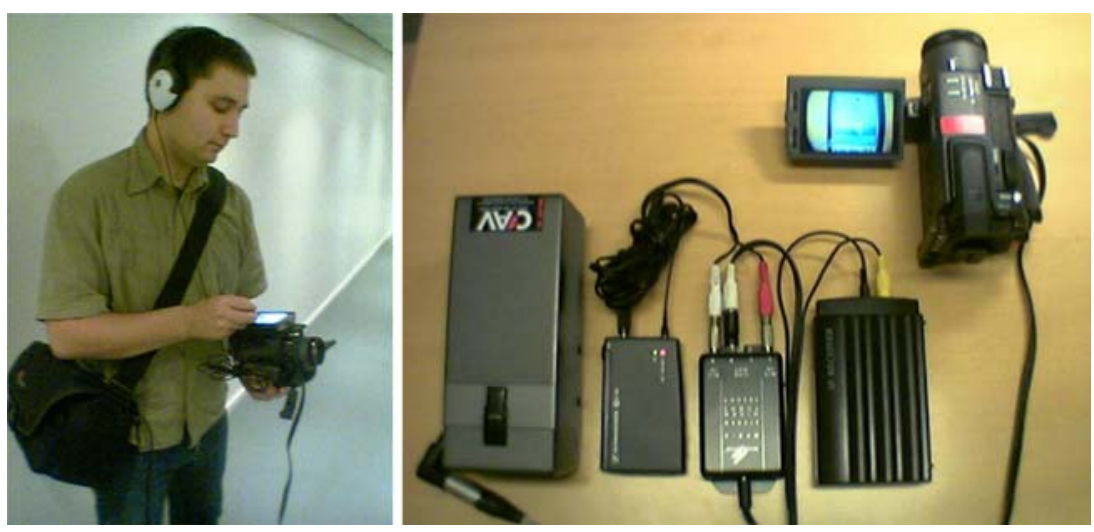

identified eight additional problems compared to the field.

Looking qualitatively at the usability problems encountered, these can be divided into three categories, concerning interaction, mobility, and contextawareness.

First, usability problems arose from nurses having problems with interacting with the system or understanding the interface. For example, one nurse did not know the semantics of the keyboard for typing in textual notes about patients. Specifically, she felt insecure about the buttons "Tab", "Caps" and "Shift" as she would expect them to be "tablets", "capsules" and "shift medication". Secondly, usability problems arose from aspects of mobility and working conditions. For example, one nurse was concerned about putting the mobile device in her pocket. She was afraid that she would accidentally click some buttons while walking and she stated that it would be impossible to carry the device in her hand at all times. Another problem related to mobility and working conditions was the fact that one nurse feared that the device could spread bacteria from patient to patient. Thus, she did not want to place the device on the patient's bedside table or on the bed. Finally, the studies revealed seven usability problems related to the context-aware element (all encountered in both conditions). These problems were primarily related to confusion among the nurses when the interface "suddenly" changed contents when, for example, walking into a ward. Typically, this would make the users either confused or annoyed - especially if reading information on the screen at the time of the automatic update. Surprisingly, however, all six, field test subjects (but only one lab subject) did not understand why the system would automatically update information and functionality according to the physical location. So, even though their use situation was in situ, and closely related to the context, they still got confused about the system being actively contextaware. Analyzing this result, we find that their reluctance towards the automatic-update element in the mobile device may stem from the consequently decreased lack of control. Operating and working in a safety-critical environment like healthcare, the decreased level of control may not appear to support systematic work practices, but merely to compromise the work activities. The feeling of lack of control is well-known to active context-aware mobile system [3] and should probably be investigated further.

In the following sub sections, we revisit the three issues of mobility, complexity and relation to work activities encountered in the study of the commercial ubiquitous EPR system in the light of the findings from our context-aware mobile counterpart. We then take a step back and discuss some general implications for ubiquitous computing emerging from our study.

\subsection{Mobility revisited}

Issues of mobility are crucial in many activities for nurses acting in a professional environment. Nurses would normally find themselves visiting patients in different physical locations and they often require different kinds of information for dependent and independent work tasks. The aspects of mobility in our study can be considered local mobility, as described in [2], and therefore the nurses would normally not require directional guidance from the system. Thus, we attempted to support the local mobility through a relatively small, handheld device that could be carried around by the nurses (potentially in their pockets) while visiting patients or conducting other work tasks. The idea of having a mobile device was appreciated by all nurses in our evaluation. However, we found that the nurses would continuously switch between reading or storing information on the device and conducting 
work tasks without the device, for example, taking measurements from patients. Thus, holding the device in their hands all the time would be impossible and therefore they would occasionally need to put it away or lay it down. This caused problems to most of the test subjects, as they did not know where to put the handheld device. As a consequence, some of them requested functionalities allowing them to lock the screen. Others questioned the general usefulness of handheld devices.

\subsection{Complexity revisited}

The first study identified another important issue with EPRs, namely, the issues concerned with complexity and fragmentation of information. Most subjects experienced problems in locating relevant and adequate information in the traditional EPR. This could be a result of many different circumstances, but one of the problems was the extensive amount of different types of information on each screen (Fig. 2). The nurses would occasionally fail to notice relevant or even critical information on, for example, patients and scheduled operations. As a result, more subjects failed to solve all assigned tasks in the study. To address this problem, we aimed at presenting much less information at a time on the mobile device by exploiting context-awareness to, for example, only presenting information about patients close by. Validating this approach, the nurses encountered no severe complexity problems when using the mobile device. However, they would occasionally request more information than could be fitted into the screen at one time.

\subsection{Relation to work activities revisited}

As a final issue from the two usability evaluations, we discovered that nurses would typically require very specific information based on current work tasks and activities. The traditional EPR did not fully support this, but presented too much, too little, or too fragmented information. In the mobile EPR prototype, we utilized context-awareness in different ways as a mean for determining the work task of the nurses. However, this also introduced some pitfalls, as nurses would sometimes miss reminders presented on the screen because their focus was engaged elsewhere. Furthermore, some nurses became confused or even annoyed by the automatic adaptation of information on the screen to their physical location. Thus, the use of context-awareness was not experienced as universally useful and further research into issues such as user control in interaction design with such systems is clearly needed.

\subsection{General implications for ubiquitous computing} in the real world

Taking a step back from the specific findings from our empirical studies at the hospital wards, a series of general implications for ubiquitous computing in the real world emerge. Overall, we find that mobile computing in the healthcare domain-whether contextaware or not- is not an alternative to the use of ubiquitous computing environment consisting of networked desktop and laptop terminals situated throughout the environment. Rather, mobile access to such ubiquitous computing system has potentials to supplement information access from stationary (and semi-mobile) terminals strategically situated in the working environment. In the real world, mobile systems are only one of many components of a truly useful ubiquitous computing system. While based on a study of specific work activities in a specific real world domain, we believe this will also apply generally to other organizations in which workers are required to be mobile within a (relatively) limited physical area while at the same time dependent on access to a large amount of shared information. Mobile access to patient record information at a hospital ward is useful for nurses in many situations because their work often require them to move between different physical locations. In these situations, the nurses usually only require very specific information related to their current physical location, task at hand or patient under treatment. Hence, handheld device automatically adapting to these contextual factors have great potentials for adding to the usefulness of the ubiquitous computing environment-provided that specific and usability issues related to context-awareness (such as user control) are carefully taken into consideration in their interaction design. At the same time, however, stationary access to patient record information via conventional computer terminals situated throughout the hospital wards is also very important. To use Kristoffersen and Ljungberg's notion of mobility [13], the mobility of the work activities in the hospital wards does not just include "wandering" from place to place but also "visiting" specific key location for longer periods of time (such as offices, consultation rooms, etc.). When accessing patient data from one of these key locations, more detailed information is often sought for, than when standing near the patient's bed-including looking through the patient's history of treatment, medication, treatment notes etc. Also, this 
is very often so, where more detailed notes and reports on treatments are entered into the system. Hence, traditional PC terminals with larger screens and better input devices for browsing information and entering text than offered by both portable (laptops) and mobile (PDAs), is by far, a preferable approach.

In summary, we found that the use of both stationary and mobile terminals at the hospital complemented each other very well in response to the three identified issues of mobility, complexity, and work relation.

Having said that the use of both stationary and mobile terminals complemented each other very well, however, we still believe that ubiquitous computing environments in the healthcare domain (and in similar domains) could be improved much further. While the combination of mobile context-aware and stationary context-independent access to the shared resource of patient information accommodated for mobile, nomadic, and stationary work, reduced complexity of information access without removing the ability to access complex information, and related patient information more closely to work activities, it is our impression that there is still a huge potential and relevance for additional technologies in between the two. In the case of the hospital, nurses and doctors are not the only ones who are mobile. So are patients, beds, and medical equipment. In fact, some of these are already often associated with highly specific situated information, which is currently not linked in with the EPR systems. Patients have wristbands with written information and sometimes carry printouts of subset of their patient record allowing not only medical staff, but also administrative staff, quick overview of, for example, upcoming medicals. Beds are equipped with printouts of information about blood pressure, temperature etc. for their associated patients, again allowing for easy (and implicitly context-related) access to key information without having to interact with a mobile device. Equipments, sometimes have written notes attached to them about how to operate them and who to contact in case of malfunction. Whereas the traditional PC terminals provide centralized access to information about all patients and the mobile context-aware terminals provide access to a subset of this information adapted to the user's context (location, etc.), information could also be provided through the environment itself in the form of, for example, situated displays located on locations, objects and people of importance.

Furthermore, we find that the use of PDAs and PCs (as well as situated displays etc.) as points of access to a ubiquitous computing system should not be seen in isolation from each other. Rather, it should be acknowledged that users of a ubiquitous computing system sometimes are most likely to use different points of access such as PDAs, PCs, etc. in combination, to solve a given task at hand, and that they will frequently shift backwards and forwards between these. In response to this, real world ubiquitous computing systems in the healthcare domain (and domains alike) should strive for seamless integration of their different elements, and allow users to apply and appropriate their combined functionality in a highly flexible manner-as also described in [2]. Information must be easily portable between devices, and it should be easy to shift from one device to another in the middle of a task without having to start over from scratch. As a simple example of this, time spent on browsing complex information hierarchies in the EPR system could be significantly limited if when having accessed information about a specific patient on your PDA, you could immediately direct other terminals, such as a PC in the office or a laptop in the ward, to the same place in the records and vise-versa.

\section{Conclusions}

We have explored the use of ubiquitous computing in the real world through a series of studies in the healthcare domain. Supporting work activities in healthcare is a highly complex and challenging task and the healthcare domain is a potential candidate for advanced ubiquitous computer systems. In response to this, we have conducted a study over two overall phases. First, we identified important challenges of supporting work tasks in healthcare through the evaluation of a ubiquitous EPR system at use at a large hospital. Second, we designed, implemented, and evaluated a mobile extension of this ubiquitous EPR system addressing identified challenges of mobility, complexity and relation to work activities by utilizing context-awareness as a key means for supporting the nurses' interaction with the EPR system. Our results show that workers in the healthcare domain can benefit from ubiquitous computing environments and that ubiquitous computer environments in the healthcare domain may be improved through mobile and contextaware points of access. However, our studies also confirm that the design of ubiquitous computing systems for the real world needs a lot of further investigations. Also, even though our findings showed that context-awareness can be applied as a useful means of exploring mobility of healthcare workers to reduce complexity of information and improve the relation between information in the EPR system and the nurses' work tasks, we found that context-awareness is a 
very difficult style of interaction to master, raising serious new challenges in relation to, for example, user control. Context-awareness has huge potentials for ubiquitous computing environments but should not be seen as a universally useful paradigm of interaction design.

The of mobile and stationary terminals in a ubiquitous computing environment compliment each other very well, when used for the types of work activities studied in this research. At the same time, however, we speculate that the usefulness of such ubiquitous computing system in the real world would increase further from additional means of information access. This could, for example, be in the form of small, situated displays located at key locations and on key objects in the working environment. In line with related research, we also speculate that the usefulness of ubiquitous computing environments comprising of an ensemble of different devices such as mobile terminals, desktop PCs, laptops and situated displays would enhance from seamless integration between these devices and services, including easy and flexible exchange of files, pointers to files, user identities etc, allowing for unforeseen use and user appropriation of ubiquitous technologies over time.

Acknowledgments The authors thank all test subjects and the Hospital of Frederikshavn for kind collaboration throughout the project. The usability evaluations were conducted in collaboration with Jan Stage, Benedikte Skibsted Als and Rune Thaarup Høegh. MobileWARD was designed and implemented by Rune Thaarup Høegh, Karsten Kryger Hansen and Søren Lauritsen. This research is supported by the Danish Technical Research Council (projects 26-04-0026 and 2106-04-0022).

\section{References}

1. Arshad U, Mascolo C, Mellor M (2003) Exploiting mobile computing in healthcare. In: Proceedings of demo session of the 3rd international workshop on smart appliances, ICDCS03

2. Bardram J, Kjær T, Nielsen C (2003) Supporting local mobility in healthcare by application roaming among heterogeneous devices. In: Proceedings of mobile HCI'03, Springer, Berlin Heidelberg New York (LNCS), pp 161-176

3. Barkhuus L, Dey A (2003) Is context-aware computing taking control away from the user? Three levels of interactivity examined. In: Proceedings of the 5th international conference on ubiquitous computing, Springer, Berlin Heidelberg New York (LNCS), pp 149-156

4. Brinck T, York G (1998) User interfaces for computer-based patient records. In Proceedings of CHI'98, New York, ACM

5. Consolvo S, Arnstein L, Robert-Franza B (2002) User study techniques in the design and evaluation of a ubicomp environment. In: Proceedings of the 4th international conference on ubiquitous computing, Springer, Berlin Heidelberg New York (LNCS), pp 73-90

6. Crabtree A, Rodden T, Hemmings T, Benford B (2003) Finding a place for ubiComp in the home. In: Proceedings of the 5th international conference on ubiquitous computing, Springer, Berlin Heidelberg New York (LNCS), pp 208-226

7. Green N, Harper R, Murtagh G, Cooper G (2001) Configuring the mobile user: sociological and industry views. Pers Ubiquitous Comput 5(2):146-156

8. Grimson J, Grimson W (2000) The SI Challenge in Health Care. Commun ACM 43(6):49-55

9. Hansen KK, Høegh RT, Lauritsen S (2003) Making contextawareness (at) work, Aalborg University, Aalborg

10. Høegh RT, Skov MB (2004) Exploring context-awareness as mean for supporting mobile work at a hospital ward. In: Proceedings of the 7 th international conference on work with computing systems (WWCS 2004)

11. Kaplan SM, Fitzpatrick G (1997) Designing support for remote intensive-care telehealth using the locales framework. In: Proceedings of DIS'97, ACM

12. Kjeldskov J, Skov MB, Stage J (2002) Usability evaluation of IBM IPJ 2.3 electronic patient record, Aalborg University, Aalborg

13. Kristoffersen S, Ljungberg F (1999) Mobile use of IT. In Proceedings of the 19th information systems research seminar in Scandinavia. Finland

14. Molich R (2000) Usable web design (In Danish). Ingeniøren I bøger

15. Morton S, Bukhres O (1997) Utilizing mobile computing in the Wishard Memorial Hospital ambulatory service. In: Proceedings of SAC'97, ACM

16. Nielsen J (1993) Usability engineering, Morgan Kaufmann

17. Rindfleish TC (1997) Privacy, information technology, and health care. Commun ACM 40(8):93-100

18. Rubin J (1994) Handbook of usability testing, Wiley

19. Schilit BN, Theimer MM (1994) Disseminating active map information to mobile hosts. IEEE Netw 8(5):22-32

20. Skov MB (2002) Design of interactive narratives: concepts, methods, and architectures. Aalborg University, Aalborg

21. Skov MB, Høegh RT (2006) Supporting information access in a hospital ward by a context-aware mobile electronic patient record. In: Crestani $\mathrm{F}$ et al (eds) Personal and ubiquitous computing, Springer, Berlin Heidelberg New York, 10(4):205-214

22. Urban M, Kunath M (2002) Design, deployment and evaluation of a clinical information system which uses mobile computers and workstations. In: Proceedings of the 2nd conference on mobiles computing in der Medizin, Heidelberg 